\title{
The Association Between the Perceived Unjust Treatment in Healthcare Settings and the Unmet Need for General Practitioner Services Among People With Foreign Background in Finland
}

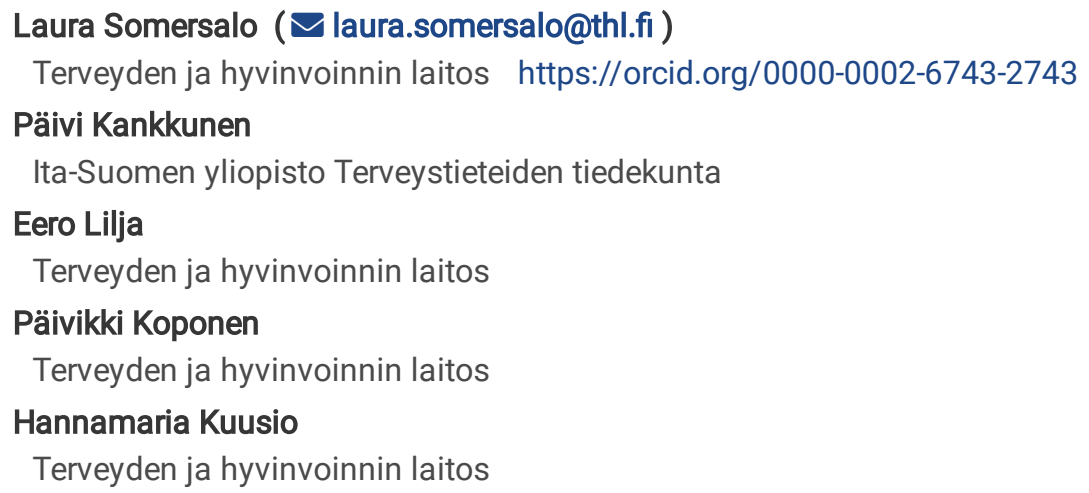




\section{Abstract}

Background: Unjust experiences are relatively common among people with foreign background (PFB) in Finland. Despite universal access to public health care, previous studies have shown inequities in the unmet need for medical care between immigrants and the general population. This study examines the association between unjust treatment in healthcare settings and unmet need for general practitioner (GP) services among PFB.

Method: The data for this study were drawn from Survey on work and well-being among people of foreign origin (UTH) $(n=4977$, response rate $66 \%$ ). The respondent characteristics were weighted and summarized, and multivariate logistic regression analyses were performed to assess the adjusted odds ratios (OR) of association between perceived unjust treatment and unmet need for GP services. The analyses were conducted in a four-step process where the first model tested the association between unjust treatment in health care settings and unmet need for GP services, second model adjusted this association by sociodemographic factors, third model was further adjusted by migration related factors, and the fourth model adjusted the previous models even further by health related factors.

Results: The results of multivariate regression showed that PFB reporting unjust treatment were also significantly more likely to report an unmet need for GP services. The difference remained significant even after controlling for other tested factors $(\mathrm{OR}=8.68,95 \% \mathrm{Cl} 6.09$ $12.36, p<.001)$. In addition to perceived unjust treatment, only younger age, lower self-rated health and existing long-term illness were significantly associated with unmet need for medical care in the final, fully adjusted model.

Conclusions: Thus, perceived unjust treatment in health care settings is significantly associated with unmet need for general practitioner services. Ensuring cultural competence throughout the entire organizational structures creates an environment to promote equal treatment for all clients. The overall costs can be reduced effectively by giving the best possible treatment for all health care users.

\section{Introduction}

Despite universal health coverage, previous studies have shown that in many countries there are inequities in unmet need for medical care between people of foreign background (PFB) and the general population. ${ }^{1-3}$ Similar results have been reported in Finland ${ }^{4,5}$. The experiences of unjust treatment appear to be more common among immigrants and ethnic minorities in comparison to the general population in many countries ${ }^{6,7}$, including Finland ${ }^{8}$.

For the past decade, the number of PFB has been increasing in Finland each year. In 2018, the number of PFB living in Finland was around 402,000 that corresponds to about 7.3 percent of the entire Finnish population. The three most common countries of origin among PFB were Russia or former Soviet Union, Estonia and Iraq. Around $46 \%$ of the PFB had moved to Finland because of family or love, whereas $19 \%$ had moved to Finland for work related reasons and $15 \%$ to seek refuge ${ }^{9}$. Approximately 49 percent of the PFB were living in the Helsinki Metropolitan Area that includes three of the four biggest cities in Finland ${ }^{10}$. In addition, most of the PFB living outside the Helsinki Metropolitan Area also tend to live near big cities ${ }^{11}$.

In Finland, the healthcare system consists of public, private and occupational healthcare providers. All of these provide clients with general practitioner (GP) services at health care centers and hospitals ${ }^{12}$. Home visits by GPs are not common and compared to many other European countries, nurses have a relatively strong role in the Finnish primary health care system ${ }^{13}$. The public health care sector is universal and comprehensive. The users pay a relatively small client fee for the public services. However, the waiting times are often long. The private sector often charges higher client fees that may exclude potential clients, but often the waiting times are much shorter than those in the public sector. In addition to public and private health care, the employed are entitled to free occupational health services. By law, the occupational health care covers preventive care, but often it also includes curative care ${ }^{14}$. Thus, the employed often have better access to primary health care services compared to the unemployed ${ }^{12}$

Previous studies have shown that PFB often experience barriers in accessing and utilizing medical care services. The barriers can be internal, structural or related to social assimilation, and are often complex and multilayered. ${ }^{15}$ Previous research evidence has shown that cultural differences, linguistic difficulties, obstacles in navigating a foreign health care system, different understandings of illness and treatment, potential negative attitudes among staff and other patients, economic challenges and lack of access to medical history can all act as barriers in accessing medical care ${ }^{5,15,16}$. Addressing just one barrier is often insufficient in gaining better access to medical care. Instead, a more holistic approach is needed. For example, the fact that a foreigner speaks the language of their new 
country of residence does not alone guarantee adequate access to health services, if the differences in health service structures between the two countries are too great to overcome ${ }^{17}$.

Discriminatory experiences can be defined as any unequal or unjust treatment of individuals or a specific group ${ }^{18}$. The relationship between the experiences of unjust treatment and health has been studied extensively, and the experiences of unjust treatment have been found to be strongly associated particularly with mental health problems ${ }^{19,20}$. Despite the existence of contradicting studies ${ }^{21}$, discrimination has been linked to an increased likelihood of somatic and chronic illnesses ${ }^{20,22}$. Perceived unjust treatment has also been linked to poorer self-rated health ${ }^{8,20}$ which is a widely accepted predictor of both morbidity and mortality ${ }^{23}$. Those that report poor self-rated health or have existing chronic illnesses often also express greater need for medical care than others ${ }^{3,24}$.

Furthermore, the experience of unjust treatment has been found to be associated with more negative patient experiences, lack of trust in and communication with the service provider, lower service satisfaction, delays in accessing services and poorer commitment to care. ${ }^{6}$ A study on the use of cross-border health care services by Russians living in Finland found that perceived unjust treatment in medical care settings increased the likelihood of cross-border healthcare usage. By contrast, a higher degree of social integration predicted less use of cross-border medical services. ${ }^{25}$ Age at migration has been recognized as a moderator of social integration as it affects different opportunities as well as ability to and efficiency in learning new languages ${ }^{26,27}$.

The need for medical care can be assessed from an objective, health care provider's viewpoint as well as from a more subjective, health care user's own viewpoint. The subjective need is perceived and sometimes expressed, and can vary greatly between different cultures and geographical locations. ${ }^{28}$ It might also differ strongly from the objective view of need for care ${ }^{16}$. This study focuses on the subjective and self-perceived need for GP services.

A large international review study suggested that PFB have a lower tendency of using medical services than the general population. Variation between different groups of PFB was also found in the study. ${ }^{29}$ PFB that have experienced unjust treatment in medical settings often have fewer medical visits, particularly in relation to preventive care ${ }^{1,30}$, and lower attendance to health screenings ${ }^{31}$. A study about childhood cancer mortality and survival among immigrants in Finland showed that patients of foreign background have higher mortality rates compared to those of Finnish background. ${ }^{5}$

Although unmet need for GP care and experiences of unjust treatment in medical settings have been studied separately, their association has not been sufficiently examined. The aim of this study is to investigate the association between perceived unjust treatment in health care settings and unmet need for GP services among PFB in Finland, and to see whether factors, such as gender, age, highest received education, employment status, marital status, residence area, age at migration, reason for migration, years lived in Finland, level of spoken Finnish or Swedish, self-rated health and existing long-term illness adjust this association.

\section{Methods}

\section{Sample}

The data used in this study are from cross-sectional Survey on work and well-being among people of foreign origin (UTH $\left.{ }^{32}\right)$, in which a total of 5,449 PFB aged between 15 and 64 were randomly selected from the Population Register Centre database in August 2013. The survey was carried out between 2014 and 2015 using face-to-face interviews in 13 different languages. After the removal of the overcoverage, the final response rate for the UTH survey was $66 \%(n=3,262)$. In this study, the data was limited to the UTH respondents who were over $18(n=3,086)$ and had answered the health-module of the survey $(n=2,689)$. Of these respondents 2,580 had answered both the question about unmet need for GP services (the outcome variable) and the question about perceived treatment in healthcare settings Only those who had used health services in the past 12 months were further analyzed using the logistic regression models $(n=$ 1,569).

The survey data were subsequently linked with the register data provided by different register databases. Age, gender, marital status, residence area, country of origin, age at migration and years lived in Finland were linked to the survey data using the register database maintained by Digital and Population Data Services Agency. The survey data of education level were obtained from Statistics Finland.

\section{Measures}

\section{Outcome variable}


Unmet need for GP was measured with the question: Do you feel that you have adequately received primary health care GP services during the past 12 months? The respondents were able to anwer using following options: ' 1 = I have not needed the service'; 2 = 'I would have needed the service but did not receive it'; 3 = 'I have received the service but it was not adequate'; and 4 = 'I have received the service and it was adequate'. In this study, a new binary variable 'unmet need for GP services' was formed by combining options 2 and 3 as 'yes' and assigning option 4 the value 'no'. Those who had not needed the service were excluded from the analyses.

Perceived unjust treatment in health care was assessed with the question: 'Have you used the following services, or had contacts with these authorities during the past 12 months? If you have, how have you been treated by them? A health center or other type of health service (e.g. doctor, nurse or other health professional at a health center, doctor's practice, or hospital)?' The options for answering were: 1 = 'I have not used the service'; 2 = 'I have used the service, and I was treated well'; and 3 = 'I have used the service, and I was treated unjust'. In this study, a new binary variable 'Unjust treatment in medical services' was formed by assigning option 2 the value 'yes' and option 3 the value 'no'. Those who had not used the services were excluded from the analyses.

\section{Sociodemographic factors}

Potential sociodemographic factors included age $(0=18-41$ years old; $1=42-64$ years old $)$, legal gender $(1=$ male, $2=$ female $)$, highest obtained education ( $1=$ primary level; $2=$ secondary level; $3=$ higher level), employment status, marital status $(1=$ married or domestic partnership; 2 = others) and residence area (1 = Capital Region; 2 = Other parts of Finland).

Self-reported employment status was categorized into two groups: 0 = 'employed' and 1 = 'unemployed and others'.

\section{Migration-related factors}

Potential migration related factors included country of origin, age at migration ( $1=17$ years or less (including those that were born in Finland); $2=18$ years or more), reason for migration, level of spoken Finnish or Swedish and years lived in Finland $(0=$ less than 10 years; $1=10$ years or more).

In the UTH survey, the respondents were categorized into seven groups based on their country of origin: 'Russia and former Soviet Union, 'Estonia', 'Middle East and North Africa', 'Other parts of Africa', 'India, Vietnam, Thailand and other parts of Asia', 'EU- and EFTAcountries and North America' and 'Latin America, former Yugoslavia and other countries'. The categorization was based on cultural, linguistic and geographical similarities between the countries, and the comparability of the sizes of the country group was also taken into account. ${ }^{32}$ To ensure adequate cell sizes, the last two categories ('EU- and EFTA-countries and North America' and 'Latin America, former Yugoslavia and other countries') were further combined into one item named 'Other countries' in this study. From this point forward, the group 'Russia and Former Soviet Union' will be referred to as Russia and FSU, and the class 'India, Vietnam, Thailand and other parts of Asia' will be referred to as Asia.

Reasons for migration were categorized into 1 = 'Work or Study', 2 = 'Family or Love', 3 = 'Refuge', and 4 = 'Other'. The group 'Other' includes for instance re-migrated Ingrian Finns and various other reasons for migration that do not fall into the previous categories.

Finnish and Swedish are the two official languages of Finland. Thus, the level of spoken Finnish or Swedish was self-rated and in this study was categorized into 0 = 'beginner-level or less' and ' 1 = moderate or better'.

\section{Health-related factors}

Potential health-related factors included having long-term health problems $(0=$ no, $1=$ yes $)$, self-rated health and perceived treatment in medical care settings.

Self-rated health was measured with the question: 'How would you describe your current health status?' It was rated by a five-item Likert-scale ranging from 1 (good) to 5 (poor). A binary transformation was used to combine options 3-5 as 'moderate or lower' and options 2 and 1 as 'good or rather good'.

\section{Statistical analyses}

Multivariate logistic regression analyses were performed to test the association between perceived unjust treatment and unmet need for GP services. Weights were used in all analyses in order to take into account potential sampling bias and reduce the effect of nonresponse $^{32}$. The analyses were conducted in a four-step process where the first model tested the association between perceived unjust 
treatment in health care settings and unmet need for GP services, second model adjusted this association for sociodemographic indicators (age, gender, highest obtained education, employment status, marital status and residence area), the third model further adjusted the previous models for migration-related factors (country of origin, age at migration, reason for migration and years lived in Finland) and the final fourth model was yet further adjusted for health-related factors (long-term health problems and self-rated health). The odds ratios (OR) and corresponding $95 \%$ confidence intervals $(95 \% \mathrm{Cl})$ as well as the coefficient of determination of all models were calculated. Possible moderation properties of all factors were tested with a model that included the two main effects and their interaction terms. All analyses were performed using the SAS 9.4 software.

\section{Results}

More than half of male respondents (56\%) and more than two thirds of female respondents (70\%) had experienced need for GP services in the past 12 months (Table 1). Of those who expressed a need for GP services, $23 \%$ of male and $28 \%$ of female respondents reported unmet need for GP services while $11 \%$ of male and $14 \%$ of female respondents had experienced perceived unjust treatment in health care settings. There were no significant differences between the genders or among country groups in unmet need for GP services or perceived unjust treatment in health care services. 
Table 1

Descriptive and weighted statistics of the study population, in percentages (\%)

\begin{tabular}{|c|c|c|c|c|c|c|c|c|c|}
\hline & Male & Female & Total & $\begin{array}{l}\text { Former } \\
\text { Soviet } \\
\text { Union and } \\
\text { Russia }\end{array}$ & Estonia & $\begin{array}{l}\text { Middle } \\
\text { East and } \\
\text { North } \\
\text { Africa }\end{array}$ & $\begin{array}{l}\text { Other } \\
\text { parts } \\
\text { of } \\
\text { Africa }\end{array}$ & $\begin{array}{l}\text { India, Vietnam, } \\
\text { Thailand and } \\
\text { other parts of } \\
\text { Asia }\end{array}$ & $\begin{array}{l}\text { Other } \\
\text { countries } \\
\text { a }\end{array}$ \\
\hline & $\begin{array}{l}n= \\
1173\end{array}$ & $\begin{array}{l}n= \\
1407\end{array}$ & $\begin{array}{l}n= \\
2580\end{array}$ & $\mathrm{n}=712$ & $\mathrm{n}=320$ & $\mathrm{n}=347$ & $\begin{array}{l}n= \\
227\end{array}$ & $\mathrm{n}=417$ & $\mathrm{n}=557$ \\
\hline Need for GP services & $\begin{array}{l}55.8 \\
(52.6- \\
59.0)\end{array}$ & $\begin{array}{l}69.9 \\
(67.3- \\
72.4)\end{array}$ & $\begin{array}{l}62.9 \\
(60.8- \\
64.9)\end{array}$ & $\begin{array}{l}63.6 \\
(59.6- \\
67.4)\end{array}$ & $\begin{array}{l}61.8 \\
(55.9- \\
67.4)\end{array}$ & $\begin{array}{l}69.8 \\
(64.1- \\
75.0)\end{array}$ & $\begin{array}{l}67.4 \\
(60.4- \\
73.7)\end{array}$ & $\begin{array}{l}62.2(57.1- \\
67.0)\end{array}$ & $\begin{array}{l}58.7 \\
(54.1- \\
63.1)\end{array}$ \\
\hline \multicolumn{10}{|c|}{ Respondents who needed GP services } \\
\hline & $\begin{array}{l}n= \\
630\end{array}$ & $\mathrm{n}=939$ & $\begin{array}{l}n= \\
1569\end{array}$ & $\mathrm{n}=449$ & $\mathrm{n}=187$ & $\mathrm{n}=240$ & $\begin{array}{l}n= \\
146\end{array}$ & $n=239$ & $n=308$ \\
\hline $\begin{array}{l}\text { Unmet need for GP } \\
\text { services }\end{array}$ & $\begin{array}{l}23.4 \\
(19.9- \\
27.3)\end{array}$ & $\begin{array}{l}28.2 \\
(25.3- \\
31.4)\end{array}$ & $\begin{array}{l}26.1 \\
(23.8- \\
28.6)\end{array}$ & $\begin{array}{l}28.8 \\
(24.6- \\
33.3)\end{array}$ & $\begin{array}{l}24.4 \\
(18.5- \\
31.4)\end{array}$ & $\begin{array}{l}30.9 \\
(25.1- \\
37.5)\end{array}$ & $\begin{array}{l}22.5 \\
(15.9- \\
30.9)\end{array}$ & $\begin{array}{l}25.7(20.1- \\
32.2)\end{array}$ & $\begin{array}{l}23.5 \\
(18.7- \\
29.1)\end{array}$ \\
\hline $\begin{array}{l}\text { Unjust treatment in } \\
\text { medical services }\end{array}$ & $\begin{array}{l}11.3 \\
(9.0- \\
14.1)\end{array}$ & $\begin{array}{l}14.4 \\
(12.1- \\
17.0)\end{array}$ & $\begin{array}{l}13.0 \\
(11.4- \\
14.9)\end{array}$ & $\begin{array}{l}11.3(8.5- \\
14.7)\end{array}$ & $\begin{array}{l}12.9 \\
(8.6- \\
18.8)\end{array}$ & $\begin{array}{l}15.9 \\
(11.7- \\
21.2)\end{array}$ & $\begin{array}{l}16.2 \\
(10.5- \\
24.1)\end{array}$ & $\begin{array}{l}13.9(10.1- \\
18.8)\end{array}$ & $\begin{array}{l}11.6 \\
(8.3- \\
16.0)\end{array}$ \\
\hline \multicolumn{10}{|l|}{ Age (in years) } \\
\hline $18-41$ & $\begin{array}{l}68.3 \\
(64.1- \\
72.3)\end{array}$ & $\begin{array}{l}65.8 \\
(62.5- \\
68.9)\end{array}$ & $\begin{array}{l}66.9 \\
(64.3- \\
69.4)\end{array}$ & $\begin{array}{l}52.4 \\
(47.5- \\
57.3)\end{array}$ & $\begin{array}{l}66.5 \\
(58.6- \\
73.6)\end{array}$ & $\begin{array}{l}71.3 \\
(65.1- \\
76.8)\end{array}$ & $\begin{array}{l}68.8 \\
(59.5- \\
76.8)\end{array}$ & $\begin{array}{l}76.9(70.9- \\
82.0)\end{array}$ & $\begin{array}{l}71.8 \\
(66.4- \\
76.7)\end{array}$ \\
\hline $42-64$ & $\begin{array}{l}31.7 \\
(27.7- \\
35.9)\end{array}$ & $\begin{array}{l}34.2 \\
(31.1- \\
37.5)\end{array}$ & $\begin{array}{l}33.1 \\
(30.6- \\
35.7)\end{array}$ & $\begin{array}{l}47.6 \\
(42.7- \\
52.5)\end{array}$ & $\begin{array}{l}33.5 \\
(26.4- \\
41.4)\end{array}$ & $\begin{array}{l}28.7 \\
(23.2- \\
34.9)\end{array}$ & $\begin{array}{l}31.2 \\
(23.2- \\
40.5)\end{array}$ & $\begin{array}{l}23.1(18.0- \\
29.1)\end{array}$ & $\begin{array}{l}28.2 \\
(23.3- \\
33.6)\end{array}$ \\
\hline \multicolumn{10}{|l|}{$\begin{array}{l}\text { Highest received } \\
\text { education }\end{array}$} \\
\hline Primary & $\begin{array}{l}20.6 \\
(17.4- \\
24.4)\end{array}$ & $\begin{array}{l}19.6 \\
(17.0- \\
22.5)\end{array}$ & $\begin{array}{l}20.1 \\
(18.0- \\
22.3)\end{array}$ & $\begin{array}{l}7.6(5.4- \\
10.6)\end{array}$ & $\begin{array}{l}20.6 \\
(15.0- \\
27.6)\end{array}$ & $\begin{array}{l}39.0 \\
(32.6- \\
45.8)\end{array}$ & $\begin{array}{l}37.9 \\
(29.5- \\
47.0)\end{array}$ & $\begin{array}{l}24.9(19.7- \\
31.0)\end{array}$ & $\begin{array}{l}11.8 \\
(8.2- \\
16.7)\end{array}$ \\
\hline Secondary & $\begin{array}{l}44.4 \\
(40.1- \\
48.8)\end{array}$ & $\begin{array}{l}41.0 \\
(37.7- \\
44.4)\end{array}$ & $\begin{array}{l}42.5 \\
(39.8- \\
45.2)\end{array}$ & $\begin{array}{l}47.0 \\
(42.1- \\
51.9)\end{array}$ & $\begin{array}{l}61.9 \\
(54.3- \\
69.0)\end{array}$ & $\begin{array}{l}34.5 \\
(28.5- \\
41.0)\end{array}$ & $\begin{array}{l}38.0 \\
(29.5- \\
47.4)\end{array}$ & $\begin{array}{l}34.4(28.1- \\
41.3)\end{array}$ & $\begin{array}{l}38.6 \\
(32.9- \\
44.7)\end{array}$ \\
\hline Higher & $\begin{array}{l}34.9 \\
(30.9- \\
39.2)\end{array}$ & $\begin{array}{l}39.4 \\
(36.1- \\
42.7)\end{array}$ & $\begin{array}{l}37.4 \\
(34.9- \\
40.1)\end{array}$ & $\begin{array}{l}45.4 \\
(40.6- \\
50.3)\end{array}$ & $\begin{array}{l}17.5 \\
(12.7- \\
23.6)\end{array}$ & $\begin{array}{l}26.5 \\
(21.0- \\
32.8)\end{array}$ & $\begin{array}{l}24.1 \\
(17.7- \\
31.8)\end{array}$ & $\begin{array}{l}40.7(34.3- \\
47.5)\end{array}$ & $\begin{array}{l}49.6 \\
(43.5- \\
55.7)\end{array}$ \\
\hline $\begin{array}{l}\text { Unemployed and } \\
\text { Others }{ }_{b}\end{array}$ & $\begin{array}{l}33.0 \\
(29.1- \\
37.1)\end{array}$ & $\begin{array}{l}48.8 \\
(45.4- \\
52.2)\end{array}$ & $\begin{array}{l}41.9 \\
(39.3- \\
44.6)\end{array}$ & $\begin{array}{l}42.2 \\
(37.5- \\
47.0)\end{array}$ & $\begin{array}{l}32.8 \\
(26.1- \\
40.2)\end{array}$ & $\begin{array}{l}60.5 \\
(53.7- \\
66.9)\end{array}$ & $\begin{array}{l}61.4 \\
(52.4- \\
69.6)\end{array}$ & $\begin{array}{l}35.4(29.4- \\
42.0)\end{array}$ & $\begin{array}{l}32.9 \\
(27.5- \\
38.9)\end{array}$ \\
\hline $\begin{array}{l}\text { Married or in a } \\
\text { Domestic } \\
\text { Partnership }\end{array}$ & $\begin{array}{l}68.1 \\
(63.8- \\
72.2)\end{array}$ & $\begin{array}{l}67.5 \\
(64.1- \\
70.7)\end{array}$ & $\begin{array}{l}67.8 \\
(65.1- \\
70.3)\end{array}$ & $\begin{array}{l}65.9 \\
(61.0- \\
70.4)\end{array}$ & $\begin{array}{l}66.6 \\
(58.5- \\
73.8)\end{array}$ & $\begin{array}{l}70.7 \\
(64.1- \\
76.5)\end{array}$ & $\begin{array}{l}54.5 \\
(45.2- \\
63.4)\end{array}$ & $\begin{array}{l}74.2(67.8- \\
79.6)\end{array}$ & $\begin{array}{l}69.8 \\
(63.7- \\
75.3)\end{array}$ \\
\hline \multicolumn{10}{|l|}{ Residence area } \\
\hline Capital Region & $\begin{array}{l}49.1 \\
(44.7- \\
53.5)\end{array}$ & $\begin{array}{l}48.0 \\
(44.6- \\
51.4)\end{array}$ & $\begin{array}{l}48.5 \\
(45.7- \\
51.2)\end{array}$ & $\begin{array}{l}38.6 \\
(33.9- \\
43.5)\end{array}$ & $\begin{array}{l}55.3 \\
(47.7- \\
62.7)\end{array}$ & $\begin{array}{l}43.8 \\
(37.1- \\
50.6)\end{array}$ & $\begin{array}{l}65.3 \\
(56.7- \\
73.1)\end{array}$ & $\begin{array}{l}55.5(48.8- \\
62.1)\end{array}$ & $\begin{array}{l}46.2 \\
(40.1- \\
52.4)\end{array}$ \\
\hline Rest of Finland & $\begin{array}{l}50.9 \\
(46.5- \\
55.3)\end{array}$ & $\begin{array}{l}52.0 \\
(48.6- \\
55.4)\end{array}$ & $\begin{array}{l}51.5 \\
(48.8- \\
54.3)\end{array}$ & $\begin{array}{l}61.4 \\
(56.5- \\
66.1)\end{array}$ & $\begin{array}{l}44.7 \\
(37.3- \\
52.3)\end{array}$ & $\begin{array}{l}56.2 \\
(49.4- \\
62.9)\end{array}$ & $\begin{array}{l}34.7 \\
(26.9- \\
43.3)\end{array}$ & $\begin{array}{l}44.5(37.9- \\
51.2)\end{array}$ & $\begin{array}{l}53.8 \\
(47.6- \\
59.9)\end{array}$ \\
\hline $\begin{array}{l}\text { Age at Migration < } \\
18 \text { years old }\end{array}$ & $\begin{array}{l}13.4 \\
(10.8- \\
16.5)\end{array}$ & $\begin{array}{l}18.6 \\
(16.0- \\
21.6)\end{array}$ & $\begin{array}{l}16.4 \\
(14.4- \\
18.5)\end{array}$ & $\begin{array}{l}22.5 \\
(18.6- \\
26.8)\end{array}$ & $\begin{array}{l}10.2 \\
(6.4- \\
15.9)\end{array}$ & $\begin{array}{l}15.8 \\
(11.5- \\
21.3)\end{array}$ & $\begin{array}{l}23.2 \\
(16.2- \\
32.1)\end{array}$ & $13.2(9.3-18.4)$ & $\begin{array}{l}13.5 \\
(9.7- \\
18.5)\end{array}$ \\
\hline
\end{tabular}




\begin{tabular}{|c|c|c|c|c|c|c|c|c|c|}
\hline & Male & Female & Total & $\begin{array}{l}\text { Former } \\
\text { Soviet } \\
\text { Union and } \\
\text { Russia }\end{array}$ & Estonia & $\begin{array}{l}\text { Middle } \\
\text { East and } \\
\text { North } \\
\text { Africa }\end{array}$ & $\begin{array}{l}\text { Other } \\
\text { parts } \\
\text { of } \\
\text { Africa }\end{array}$ & $\begin{array}{l}\text { India, Vietnam, } \\
\text { Thailand and } \\
\text { other parts of } \\
\text { Asia }\end{array}$ & $\begin{array}{l}\text { Other } \\
\text { countries } \\
\text { a }\end{array}$ \\
\hline \multicolumn{10}{|l|}{$\begin{array}{l}\text { Reason for } \\
\text { Migration }\end{array}$} \\
\hline Work or Study & $\begin{array}{l}37.6 \\
(33.3- \\
42.1)\end{array}$ & $\begin{array}{l}16.4 \\
(14.0- \\
19.2)\end{array}$ & $\begin{array}{l}25.6 \\
(23.2- \\
28.2)\end{array}$ & $\begin{array}{l}17.3 \\
(13.8- \\
21.5)\end{array}$ & $\begin{array}{l}43.6 \\
(36- \\
51.5)\end{array}$ & $\begin{array}{l}15.3 \\
(10.8- \\
21.1)\end{array}$ & $\begin{array}{l}16.4 \\
(11.2- \\
23.5)\end{array}$ & $\begin{array}{l}33.8(27.4- \\
40.9)\end{array}$ & $\begin{array}{l}28.2 \\
(22.8- \\
34.2)\end{array}$ \\
\hline Family or Love & $\begin{array}{l}38.9 \\
(34.8- \\
43.2)\end{array}$ & $\begin{array}{l}67.2 \\
(63.9- \\
70.4)\end{array}$ & $\begin{array}{l}54.9 \\
(52.1- \\
57.6)\end{array}$ & $\begin{array}{l}69.8 \\
(65.1- \\
74.1)\end{array}$ & $\begin{array}{l}44.6 \\
(37.1- \\
52.3)\end{array}$ & $\begin{array}{l}38.4 \\
(32.1- \\
45.2)\end{array}$ & $\begin{array}{l}46.6 \\
(37.8- \\
55.7)\end{array}$ & $\begin{array}{l}56.4(49.5- \\
63.2)\end{array}$ & $\begin{array}{l}57.5 \\
(51.3- \\
63.5)\end{array}$ \\
\hline Refuge & $\begin{array}{l}13.8 \\
(11- \\
17)\end{array}$ & $\begin{array}{l}8.7 \\
(7.0- \\
10.8)\end{array}$ & $\begin{array}{l}10.9 \\
(9.3- \\
12.7)\end{array}$ & $\begin{array}{l}1.8(0.9- \\
3.6)\end{array}$ & NA & $\begin{array}{l}38.9 \\
(32.7- \\
45.6)\end{array}$ & $\begin{array}{l}30.7 \\
(22.6- \\
40.2)\end{array}$ & $6.1(3.5-10.3)$ & $\begin{array}{l}5.5(3.2- \\
9.4)\end{array}$ \\
\hline Other $_{c}$ & $\begin{array}{l}9.8 \\
(7.6- \\
12.5)\end{array}$ & $\begin{array}{l}7.6 \\
(5.9- \\
9.7)\end{array}$ & $\begin{array}{l}8.6 \\
(7.2- \\
10.2)\end{array}$ & $\begin{array}{l}11.1(8.4- \\
14.4)\end{array}$ & $\begin{array}{l}11.9 \\
(7.9- \\
17.3)\end{array}$ & $\begin{array}{l}7.3(4.5- \\
11.7)\end{array}$ & $\begin{array}{l}6.2 \\
(2.5- \\
14.5)\end{array}$ & $3.6(1.6-7.8)$ & $\begin{array}{l}8.8(6- \\
12.7)\end{array}$ \\
\hline $\begin{array}{l}\text { Years lived in } \\
\text { Finland }<10\end{array}$ & $\begin{array}{l}53.2 \\
(48.8- \\
57.5)\end{array}$ & $\begin{array}{l}46.4 \\
(43- \\
49.8)\end{array}$ & $\begin{array}{l}49.3 \\
(46.6- \\
52.0)\end{array}$ & $\begin{array}{l}33.6 \\
(29.3- \\
38.3)\end{array}$ & $\begin{array}{l}65.4 \\
(57.9- \\
72.2)\end{array}$ & $\begin{array}{l}50.9 \\
(44.2- \\
57.5)\end{array}$ & $\begin{array}{l}46.3 \\
(37.6- \\
55.3)\end{array}$ & $\begin{array}{l}62.1(55.4- \\
68.4)\end{array}$ & $\begin{array}{l}48.3 \\
(42.2- \\
54.5)\end{array}$ \\
\hline $\begin{array}{l}\text { Level of spoken } \\
\text { Finnish or Swedish } \\
\text { moderate or better }\end{array}$ & $\begin{array}{l}71.0 \\
(66.8- \\
74.9)\end{array}$ & $\begin{array}{l}77.3 \\
(74.2- \\
80.1)\end{array}$ & $\begin{array}{l}74.5 \\
(72.0- \\
76.9)\end{array}$ & $\begin{array}{l}85.8 \\
(82.1- \\
88.8)\end{array}$ & $\begin{array}{l}90.2 \\
(84.6- \\
93.9)\end{array}$ & $\begin{array}{l}67.5 \\
(60.9- \\
73.5)\end{array}$ & $\begin{array}{l}69.0 \\
(60.1- \\
76.6)\end{array}$ & $\begin{array}{l}57.9(50.9- \\
64.6)\end{array}$ & $\begin{array}{l}70.8 \\
(64.6- \\
76.3)\end{array}$ \\
\hline $\begin{array}{l}\text { Self-rated health } \\
\text { moderate or lower }\end{array}$ & $\begin{array}{l}22.3 \\
(18.8- \\
26.1)\end{array}$ & $\begin{array}{l}24.3 \\
(21.5- \\
27.4)\end{array}$ & $\begin{array}{l}23.4 \\
(21.2- \\
25.8)\end{array}$ & $\begin{array}{l}33.1 \\
(28.6- \\
37.9)\end{array}$ & $\begin{array}{l}29.8 \\
(22.9- \\
37.8)\end{array}$ & $\begin{array}{l}25.6 \\
(20.1- \\
32.0)\end{array}$ & $\begin{array}{l}16.3 \\
(11.0- \\
23.5)\end{array}$ & $\begin{array}{l}15.3(11.1- \\
20.6)\end{array}$ & $\begin{array}{l}16.5 \\
(12.5- \\
21.5)\end{array}$ \\
\hline $\begin{array}{l}\text { Having a long-term } \\
\text { illness }\end{array}$ & $\begin{array}{l}32.1 \\
(28.2- \\
36.3)\end{array}$ & $\begin{array}{l}34.5 \\
(31.4- \\
37.8)\end{array}$ & $\begin{array}{l}33.5 \\
(31.0- \\
36.1)\end{array}$ & $\begin{array}{l}42.7 \\
(37.9- \\
47.6)\end{array}$ & $\begin{array}{l}36.1 \\
(28.8- \\
44.1)\end{array}$ & $\begin{array}{l}39.6 \\
(33.3- \\
46.3)\end{array}$ & $\begin{array}{l}18.4 \\
(12.8- \\
25.7)\end{array}$ & $\begin{array}{l}22.0(16.9- \\
28.1)\end{array}$ & $\begin{array}{l}32.3 \\
(27.0- \\
38.2)\end{array}$ \\
\hline $\begin{array}{l}\text { a Includes all count } \\
\text { b Includes e.g. stud } \\
\text { c Includes re-migrat } \\
\text { NA = cell size }<5\end{array}$ & $\begin{array}{l}\text { that are } \\
\text { and hol } \\
\text { ngrian } \mathrm{F}\end{array}$ & $\begin{array}{l}\text { ot part of } \\
\text { ewives } \\
\text { ns, and ot }\end{array}$ & er people & that have mo & ed to Finla & d various & fferent re & son. & \\
\hline
\end{tabular}

Of those who had needed GP services in the past 12 months $67 \%$ were less than 42 years old, $60 \%$ female, $68 \%$ married or living in a domestic partnership and $63 \%$ had at least secondary education. (Table 1.). Half of the respondents were employed (female $51 \%$, male $67 \%)$. The lowest level of employment was among people from the Middle East and Africa (39-40\%).

Almost half of the respondents (48\%) resided in the Helsinki Metropolitan Area. However, only $39 \%$ of Russia and FSU group and $65 \%$ of Other parts of Africa group resided in the Helsinki Metropolitan Area. While altogether $16 \%$ of the respondents had moved to Finland before the age of 18 , the age at migration varied among the country groups. The most common reason for moving to Finland was family- or love-related reasons (55\%) which were especially prominent among women (67\%). Seeking refuge was the most common reason for moving to Finland among people from the Middle East and Africa (30-39\%).

Around half of the respondents had lived in Finland for more than ten years. Three-fourths of the respondents assessed their level of spoken Finnish or Swedish as 'moderate or better'. This was more common among people coming from Estonia or Russia and FSU (86-90\%) and less common among people coming from Asia (58\%) than among the respondents in general (75\%).

More than three out of four of the respondents rated their health to be at least rather good. However, the proportion that rated their health at least rather good was lower among people of Russian and FSU background (67\%) and higher among people of Asian (85\%) background. Two thirds of all PFB did not suffer from any long-term illnesses. However, the prevalence of long-term illnesses was higher among people with Russian and FSU background (43\%) and lower among people in the groups 'Other parts of Africa' and 'Asia' (18$22 \%$ ) than among the PFB in general (34\%) (Table 1). 
The results of multivariate regression showed that PFB reporting unjust treatment were significantly more likely to experience unmet need for GP services (OR 8.21, CI 5.84-11.53, $p<.001$ ) (see Table 2, Model 1). After controlling the model for the sociodemographic factors, the association between unjust treatment and unmet need for GP services remained similar (OR 8.40, Cl 5.98-11.81, $p<.001)$ (see Model 2). Only employment status (OR 1.45, Cl 1.12-1.88, $p=.005)$ and higher education level $(\mathrm{OR} 1.72, \mathrm{Cl} 1.18-2.50, p=.013)$ were associated with unmet need for GP services in this model. In the third model, which was adjusted for migration-related factors, the OR between unjust treatment and unmet need for GP services increased further $(\mathrm{OR}=8.73, \mathrm{Cl} 6.18-12.33, p<.001)$. In this model, employment status was the only factor associated with GP services $(\mathrm{OR}=1.43, \mathrm{Cl} 1.08-1.89, p=.013)$. In the final model which was additionally adjusted for health-related factors, the OR between unjust treatment and unmet need for GP services decreased slightly when compared to the model $3(\mathrm{OR}=8.68, \mathrm{Cl} 6.09-12.36, p<.001)$ (see Model 4). Besides unjust treatment in medical care, younger age (OR 1.53, Cl 1.07-2.17, $p=.018)$, lower self-rated health (OR 1.44, $\mathrm{Cl} 1.02-2.02, p=.037$ ) and prevalence of long-term health problems (OR 1.66, Cl 1.22-2.287, $p=.002$ ) were associated with unmet need for GP services. 
Table 2

Unjust treatment's association with unmet need for GP services, results of logistic regression (odds ratio (OR), 95\% confidence intervals (CI), p-value, coefficient of determinations (Max-rescaled R2))

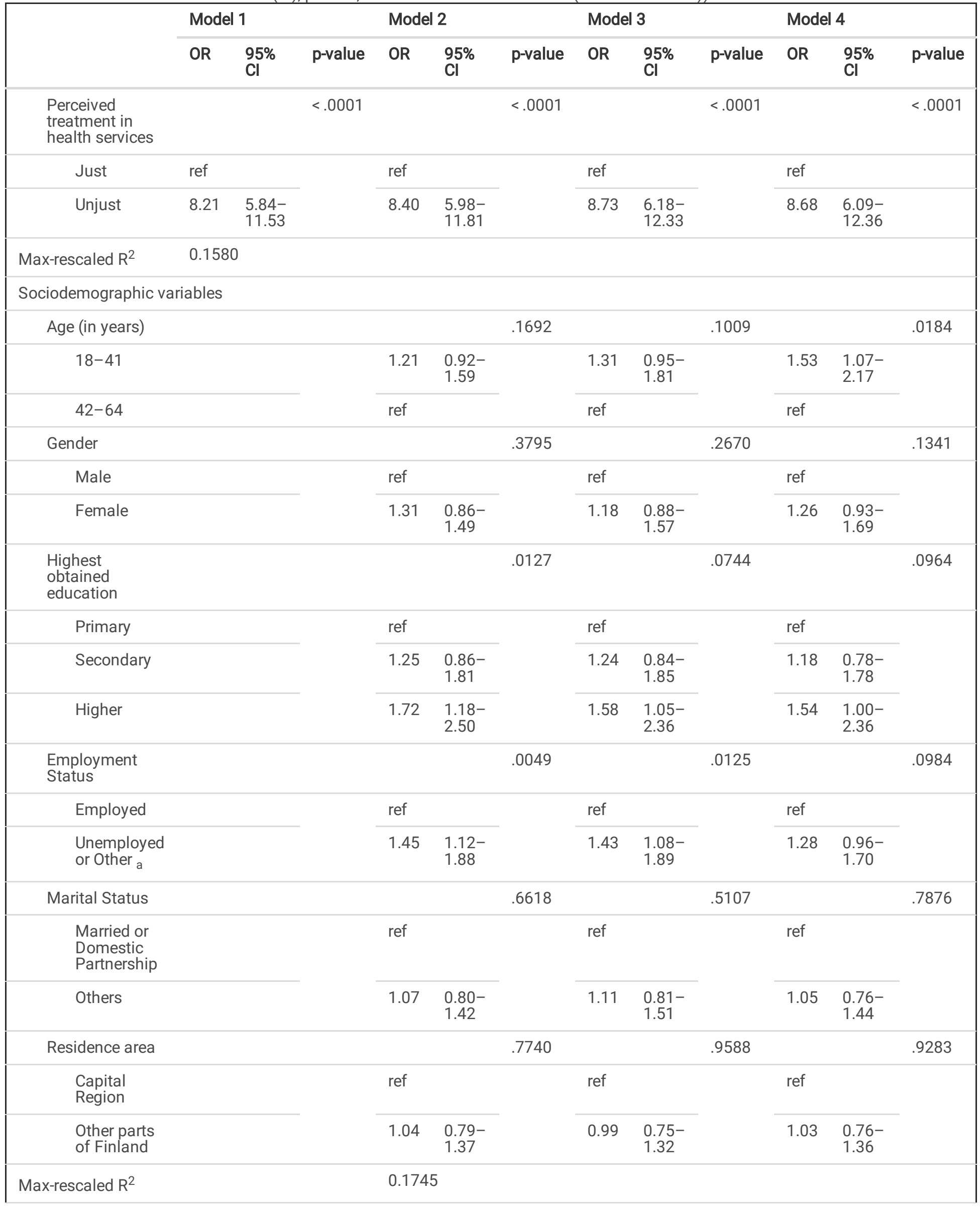




\begin{tabular}{|c|c|c|c|c|c|c|c|c|c|c|c|c|}
\hline & \multicolumn{3}{|c|}{ Model 1} & \multicolumn{3}{|c|}{ Model 2} & \multicolumn{3}{|c|}{ Model 3} & \multicolumn{3}{|c|}{ Model 4} \\
\hline & OR & $\begin{array}{l}95 \% \\
\mathrm{Cl}\end{array}$ & $\mathrm{p}$-value & OR & $\begin{array}{l}95 \% \\
\mathrm{Cl}\end{array}$ & p-value & OR & $\begin{array}{l}95 \% \\
\mathrm{Cl}\end{array}$ & p-value & OR & $\begin{array}{l}95 \% \\
\mathrm{Cl}\end{array}$ & p-value \\
\hline \multicolumn{13}{|c|}{ Migration related variables } \\
\hline $\begin{array}{l}\text { Country of } \\
\text { origin }\end{array}$ & & & & & & & & & .2041 & & & 0.655 \\
\hline $\begin{array}{l}\text { Former } \\
\text { Soviet } \\
\text { Union and } \\
\text { Russia }\end{array}$ & & & & & & & ref & & & ref & & \\
\hline Estonia & & & & & & & 0.71 & $\begin{array}{l}0.44- \\
1.15\end{array}$ & & 0.72 & $\begin{array}{l}0.44- \\
1.19\end{array}$ & \\
\hline $\begin{array}{l}\text { Middle East } \\
\text { and North } \\
\text { Africa }\end{array}$ & & & & & & & 1.61 & $\begin{array}{l}0.61- \\
1.48\end{array}$ & & 0.90 & $\begin{array}{l}0.57- \\
1.43\end{array}$ & \\
\hline $\begin{array}{l}\text { Other parts } \\
\text { of Africa }\end{array}$ & & & & & & & 0.57 & $\begin{array}{l}0.32- \\
1.01\end{array}$ & & 0.69 & $\begin{array}{l}0.38- \\
1.25\end{array}$ & \\
\hline $\begin{array}{l}\text { India, } \\
\text { Vietnam, } \\
\text { Thailand } \\
\text { and other } \\
\text { parts of } \\
\text { Asia }\end{array}$ & & & & & & & 0.78 & $\begin{array}{l}0.50- \\
1.23\end{array}$ & & 0.88 & $\begin{array}{l}0.55- \\
1.41\end{array}$ & \\
\hline $\begin{array}{l}\text { Other } \\
\text { countries }_{b}\end{array}$ & & & & & & & 0.69 & $\begin{array}{l}0.47- \\
1.03\end{array}$ & & 0.77 & $\begin{array}{l}0.51- \\
1.16\end{array}$ & \\
\hline $\begin{array}{l}\text { Age at } \\
\text { Migration }\end{array}$ & & & & & & & & & .3675 & & & 0.456 \\
\hline $\begin{array}{l}<18 \text { years } \\
\text { old }\end{array}$ & & & & & & & ref & & & ref & & \\
\hline $\begin{array}{l}\geq 18 \text { years } \\
\text { old }\end{array}$ & & & & & & & 1.25 & $\begin{array}{l}0.77- \\
2.01\end{array}$ & & 1.21 & $\begin{array}{l}0.74- \\
1.97\end{array}$ & \\
\hline $\begin{array}{l}\text { Reason for } \\
\text { Migration }\end{array}$ & & & & & & & & & .3557 & & & .3276 \\
\hline $\begin{array}{l}\text { Work or } \\
\text { Study }\end{array}$ & & & & & & & ref & & & ref & & \\
\hline $\begin{array}{l}\text { Family or } \\
\text { Love }\end{array}$ & & & & & & & 0.81 & $\begin{array}{l}0.56- \\
1.18\end{array}$ & & 0.81 & $\begin{array}{l}0.55- \\
1.19\end{array}$ & \\
\hline Refuge & & & & & & & 0.98 & $\begin{array}{l}0.56- \\
1.71\end{array}$ & & 1.06 & $\begin{array}{l}0.60- \\
1.87\end{array}$ & \\
\hline Other $_{c}$ & & & & & & & 1.22 & $\begin{array}{l}0.70- \\
2.12\end{array}$ & & 1.20 & $\begin{array}{l}0.67- \\
2.13\end{array}$ & \\
\hline $\begin{array}{l}\text { Years lived in } \\
\text { Finland }\end{array}$ & & & & & & & & & .2557 & & & .1511 \\
\hline$\leq 10$ & & & & & & & 1.21 & $\begin{array}{l}0.87- \\
1.67\end{array}$ & & 1.28 & $\begin{array}{l}0.91- \\
1.81\end{array}$ & \\
\hline$>10$ & & & & & & & ref & & & ref & & \\
\hline $\begin{array}{l}\text { Level of spoken } \\
\text { Finnish or } \\
\text { Swedish }\end{array}$ & & & & & & & & & .3516 & & & .1746 \\
\hline $\begin{array}{l}\text { Moderate or } \\
\text { higher }\end{array}$ & & & & & & & 1.20 & $\begin{array}{l}0.83- \\
1.71\end{array}$ & & 1.31 & $\begin{array}{l}0.89- \\
1.93\end{array}$ & \\
\hline
\end{tabular}

Page 10/15 


\begin{tabular}{|c|c|c|c|c|c|c|c|c|c|c|c|c|}
\hline & \multicolumn{3}{|c|}{ Model 1} & \multicolumn{3}{|c|}{ Model 2} & \multicolumn{3}{|c|}{ Model 3} & \multicolumn{3}{|c|}{ Model 4} \\
\hline & OR & $\begin{array}{l}95 \% \\
\mathrm{Cl}\end{array}$ & p-value & OR & $\begin{array}{l}95 \% \\
\mathrm{Cl}\end{array}$ & p-value & OR & $\begin{array}{l}95 \% \\
\mathrm{Cl}\end{array}$ & p-value & OR & $\begin{array}{l}95 \% \\
\mathrm{Cl}\end{array}$ & p-value \\
\hline $\begin{array}{l}\text { Beginner or } \\
\text { less }\end{array}$ & & & & & & & ref & & & ref & & \\
\hline Max-rescaled $\mathrm{R}^{2}$ & & & & & & & 0.18 & & & & & \\
\hline \multicolumn{13}{|l|}{ Health variables } \\
\hline Self-rated health & & & & & & & & & & & & .0372 \\
\hline $\begin{array}{l}\text { Good or } \\
\text { rather good }\end{array}$ & & & & & & & & & & ref & & \\
\hline $\begin{array}{l}\text { Moderate or } \\
\text { lower }\end{array}$ & & & & & & & & & & 1.44 & $\begin{array}{l}1.02- \\
2.02\end{array}$ & \\
\hline $\begin{array}{l}\text { Having long- } \\
\text { term health } \\
\text { problems }\end{array}$ & & & & & & & & & & & & .0015 \\
\hline Yes & & & & & & & & & & 1.66 & $\begin{array}{l}1.22- \\
2.27\end{array}$ & \\
\hline No & & & & & & & & & & ref & & \\
\hline Max-rescaled $\mathrm{R}^{2}$ & & & & & & & & & & 0.211 & & \\
\hline $\begin{array}{l}\text { a Includes e.g. studer } \\
\text { b Includes all countrie } \\
\text { c Includes re-migrate }\end{array}$ & $\begin{array}{l}\text { and } \mathrm{r} \\
\text { that a } \\
\text { ngriar }\end{array}$ & usewi & $\begin{array}{l}\text { of other } \mathrm{g} \\
\text { other pec }\end{array}$ & oups. & 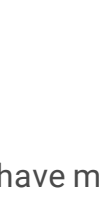 & d to Finl & ten & us diff & t reason. & & & \\
\hline
\end{tabular}

Please insert Table 2 here.

None of the tested factors moderated the association between unmet need for GP services and unjust treatment in medical care. Gender, marital status, residence area, country of origin, age at migration, reason for migration, years lived in Finland and level of spoken Finnish or Swedish did not moderate the association between unjust treatment and unmet need for care in any of the logistic regression models.

\section{Discussion}

The purpose of this study was to examine the association between perceived unjust treatment in health care settings and unmet need for GP services among PFB in Finland. The results show significant increase in odds ratio for unmet need for GP services when met with perceived unjust treatment. Adjusting for other tested factors did not affect this association. The results differ from previous studies in which the used factors have been associated with unmet need for medical care $3,5,6,12,15,16,18-22,24,26,27$. The strong association could be due to a bi-directional relationship between the discriminatory treatment and unmet need for care.

Those respondents who expressed lower self-rated health and/or existing long-term health problems were more likely to experience unmet need for care. This is in line with previous research done in Finland ${ }^{33,34}$ and in other countries ${ }^{3,30}$. In addition to health-related factors, only younger age increased the odds for unmet need for care in the final model.

Interestingly, none of the tested migration-related factors were significantly associated with unmet need for GP services. Both similar ${ }^{35}$ and contradicting ${ }^{3,33}$ results have been found in previous studies about unmet need for care among PFB. This could suggest that the reasons behind unmet need for medical care are similar among PFB and the general population of Finland. Another reason could be that the variation among different PFB groups is large, and thus the differences could not be observed in our analysis.

The data for this study are from a cross-sectional study UTH, with a response rate of $66 \%$. Even though the analysis weights were calculated with a large set of register data available for all persons in the sample, some non-response bias might still occur. Especially 
in health-related surveys, the non-respondents are often those of lower socio-economical background and with bigger health burdens ${ }^{38}$. Furthermore, the data were collected using face-to-face interviews which might cause some bias especially in connection with the most intimate and sensitive questions ${ }^{39}$. However, this has most likely caused only minor bias, as most interviewers used in the UTH survey had long experience in survey data collection and were trained to build trust with the interviewees. Other possible causes for bias in this study could include possible differences in translations or respondents' difficulties in understanding the questions.

Similar studies comparing the association of perceived treatment and unmet need for care among PFB are scarce, especially in a Finnish context, so this study makes an important contribution to the field. However, comparing the results to the general population of Finland would have given an additional perspective for the study. Unfortunately, this was not possible due to the different question formats of the surveys used for PFB and general population.

Ensuring cultural competence throughout the organizational structure, and not just as an asset in a particular employee's skillset, creates an environment that promotes equal treatment for all clients. Implementing any new practices in an organizational culture requires deliberate learning, flexibility, positive attitudes, adequate resources and training for staff as well as sufficient provision of information ${ }^{16,36,37}$. This means that organizations need to actively include cultural competence not only in their strategies, but also in their practices. One way to better meet the needs of a growingly multicultural population is to ensure diversity in healthcare personnel. This would guarantee a more diverse language skillset and cultural understanding at the healthcare setting.

It is also important to keep in mind that even though factors like level of spoken Finnish or Swedish or years lived in Finland were not significantly related to unmet need for GP services in this study, they could still affect the quality and timeliness of received services. To ensure the effectiveness of medical appointments, longer appointment times for those in need of interpreter should be made possible and appropriate information about the services available should be distributed.

In tax-funded public health care, resources are limited. By improving access to the best possible services for all health care users, the effectiveness can be increased and overall costs affected positively. This could also have an impact on both the patient's experience and their overall health.

\section{Conclusions}

Perceived unjust treatment in health care settings is significantly associated with unmet need for general practitioner services among people with foreign background in Finland. The inequities in unmet need for medical care between PFB and general population could be affected by culturally sensitive treatment and proper knowledge distribution. An environment to promote equal treatment for all clients could be created by ensuring cultural competence throughout the organizational structures.

\section{List Of Abbreviations}

GP General practitioner

PFB People with foreign background

THL Finnish Institute for Health and Welfare

UTH Survey on work and well-being among people of foreign origin

\section{Declarations}

\section{Acknowledgements}

The authors would like to thank the entire UTH survey team and the interviewers that carried out the survey, as well as the survey participants.

\section{Funding}

The work of Laura Somersalo, Eero Lilja and Hannamaria Kuusio has been supported by the European Union's Asylum, Migration and Integration Fund (AMIF). Päivikki Koponen and Päivi Kankkunen have not received any outside funding for the analysis presented here.

Page $12 / 15$ 
Author information

\section{Affiliations}

Department of Nursing Science, University of Eastern Finland, Yliopistonranta 1C, 70211 Kuopio, Finland

Laura Somersalo \& Päivi Kankkunen

Equality and Inclusion Unit, Finnish Institute for Health and Welfare, Mannerheimintie 166, 00300 Helsinki, Finland

Laura Somersalo, Hannamaria Kuusio \& Eero Lilja

Public Health Evaluation and Projection Unit, Finnish Institute for Health and Welfare, Mannerheimintie 166, 00300 Helsinki, Finland

Päivikki Koponen

\section{Contributions}

LS did all data management and analysis for this study with close supervision of HK and PKa. EL helped with guidance of analysis of data, and was involved in all stages of the study. LS prepared the first draft. All authors read and approved the final manuscript.

\section{Corresponding author}

Correspondence to Laura Somersalo.

\section{Ethics declarations}

\section{Ethics approval and consent to participate}

The UTH study was granted an ethical approval by the Institutional review board (IRB) of The Finnish Institute for Health and Welfare (THL) (Decision number: 8/2013 \$602). A consent was obtained from the respondents to both participate in the interview and to link the interview data to available register data. Respondents were assured they can stop and dropout anytime during the interview.

\section{Consent for publication}

Not applicable.

\section{Competing interests}

The authors declare that they have no competing interests.

\section{Availability of data and materials}

The data that support the findings of this study are available from Finnish Institute for Health and Welfare and Statistics Finland, but restrictions apply to the availability of these data, which were used under license for the current study, and so are not publicly available. Data are however available from the authors upon reasonable request and with permission of Finnish Institute for Health and Welfare or Statistics Finland.

\section{References}

1. Lamkaddem M, Essink-Bot ML, Deville W, Foets M, Stronks K. Perceived discrimination outside health care settings and health care utilization of turkish and moroccan GP patients in the netherlands. Eur J Public Health 2012;22(4),473-478.

2. Guidi C, Palncia L, Ferrini S, Malmusi D. Inequalities by Immigrant Status in Unmet Needs for Healthcare in Europe: The Role of Origin, Nationality and Economic Resources. European University Institute: Florence, Italy; 2016.

3. Busetta A, Cetorelli V, Wilson B. A Universal Health Care System? Unmet Need for Medical Care Among Regular and Irregular Immigrants in Italy. J Immigr Minor Health 2018;20,416-421.

4. Kuusio H, Koponen P, Castaneda AE, Lilja E, Manderbacka K, Koskinen S. Unmet need for medical care among people of foreign origin in Finland: Hannamaria Kuusio. Eur J Public Health 2016;26(suppl_1). 
5. Kyrönlahti A, Madanat-Harjuoja L, Pitkäniemi J, Rantanen M, Malila N, Taskinen M. Childhood cancer mortality and survival in immigrants: A population-based registry study in Finland. Int J Cancer 2019; Epub.

6. Ben J, Cormack D, Harris R, Paradies Y. Racism and health service utilisation: A systematic review and meta-analysis. Plos One 2017;12(12).

7. FRA. Second European Union Minorities and Discrimination Survey: Being Black in the EU. European Union Agency for Fundamental Rights. Luxembourg: Publications Office of the European Union, 2018.

8. Rask S. Diversity and health in the population: Findings on Russian, Somali and Kurdish origin populations in Finland. Eur J Public Health 2018;28(5),893-903.

9. Kuusio H, Seppänen A, Jokela S, Somersalo L, Lilja E. Survey on Well-Being among Foreign Born Population. Finnish Institute for Health and Wellbeing: 2020: http://www.julkari.fi/bitstream/handle/10024/139210/URN_ISBN_978-952-343-034-1.pdf? sequence=1\&isAllowed=y

10. Statistics Finland. Statistic Finland's PxWeb-database. The amount of migrants by regions 1990-2017: http://pxnet2.stat.fi/PXWeb/pxweb/fi/?rxid=dc815f51-a7d0-4aec-a81a-49b8ce399d18. [27.9.2019]

11. Juopperi J. Maahanmuuttajat suuntaavat kaupunkeihin - Euroopasta tulleita asettunut maaseudullekin (Immigrants move to cities - people coming from Europe also live in rural areas). Tieto \& Trendi. Statistic Finland 2019.

12. European Observatory on Health Systems and Politics. Overview of the Health System. Available from: https://www.hspm.org/countries/finland21082013/livinghit.aspx? Section=2.1\%200verview\%20of\%20the\%20health\%20system\&Type=Section [20th April 2020]

13. Ensio A, Lammintakanen J, Härkönen M, Kinnunen J. Finland. In: Rafferty AM, Busse R, Zander-Jentsch B, Sermeus W, Bruyneel L. (eds.) Strengthening health systems through nursing: Evidence from 14 European countries. Health Policy Series 52. European Observatory on Health Systems and Policies. WHO. 2019, p.34-36: https://issuu.com/whoeurope/docs/strengthening_hs_throughnursing-eng

14. Occupational Health Act (1383/2001).

15. Asgary R, Segar N. Barriers to health care access among refugee asylum seekers. J Health Care for the Poor \& Underserved 2011;22(2),506-522.

16. Priebe S, Sandhu S, Dias S, et al. Good practice in health care for migrants: views and experiences of care professionals in 16 European countries. BMC Public Health 2011;11,187

17. Akhavan $S \&$ Karlsen S. Practitioner and client explanations for disparities in health care use between migrant and non-migrant groups in Sweden: A qualitative study. J Immigr Minor Health 2013;15(1),188-197.

18. Krieger N. Embodying inequality: a review of concepts, measures, and methods for studying health consequences of discrimination. Int J Health Serv1999;29,295-352.

19. Paradies Y, Ben J, Denson N, et al. Racism as a determinant of health: A systematic review and meta-analysis. PLOS ONE 2015;10(9).

20. Wallace S, Nazroo J, Bécares L. Cumulative effect of racial discrimination on the mental health of ethnic minorities in the United Kingdom. Am J Public Health 2016;106(7),1294-3000.

21. Straiton ML, Aamb $\varnothing$ AK \& Johansen R. 2019. Perceived discrimination, health and mental health among immigrants in Norway: The role of moderating factors. BMC Public Health,19(1),325.

22. Lewis T, Cogburn C, Williams D. Self-reported experiences of discrimination and health: Scientific advances, ongoing controversies, and emerging issues. Annu Rev Clin Psychol 2015,11,407-440.

23. Idler EL, Benyamini Y. Self-rated health and mortality: a review of twenty-seven community studies. J Health Soc Behav 1997;38(1),21-37.

24. Misra R, Hunte H. Perceived discrimination and health outcomes among Asian Indians in the united states. BMC Health Serv. Res 2016;16(1),1-10.

25. Kemppainen L, Kemppainen T, Skogberg N, Kuusio H, Koponen P. Immigrants' use of health care in their country of origin: The role of social integration, discrimination and the parallel use of health care systems. Scand J Caring Sci 2018;32(2),698-706.

26. Leu J, Yen IH, Gansky SA, Walton E, Adler NE, Takeuchi DT. The association between subjective social status and mental health among Asian immigrants: investigating the influence of age at immigration. Soc Sci Med. 2008;66(5),1152-1164. 
27. Torres JM, Ro A, Sudhinaraset M. (2019). Reconsidering the Relationship between Age at Migration and Health Behaviors among US Immigrants: The Modifying Role of Continued Cross-border Ties. Advances in medical sociology, 19,17-45.

28. Asadi-Lari M, Packham C, Gray D. Need for redefining needs. Health Qual Life Outcomes 2003;1(34).

29. Sarría-Santamera A, Hijas-Gómez Al, Carmona R, Gimeno-Feliú LA. A systematic review of the use of health services by immigrants and native populations. Public Health Reviews 2016;37,28.

30. Shaaban AN, Morais S, Peleteiro B. Healthcare Services Utilization Among Migrants in Portugal: Results From the National Health Survey 2014. J Immigr Minority Health 2019;21(2),219-229.

31. Hong HC, Ferrans CE, Park C, Lee H, Quinn L, Collins EG. Effects of perceived discrimination and trust on breast cancer screening among Korean American women. Women's Health Issues 2018;28(2),188-196.

32. Nieminen T, Sutela H, Hannula U. Survey on work and well-being among people of foreign origin (UTH). Statistics Finland. 2015: https://www.stat.fi/tup/maahanmuutto/uth-tutkimus/yhteenveto_en.html

33. Koponen P, Kuusio H, Keskimäki I, et al. Unmet needs for medical care among migrants in Finland. Eur J Public Health 2014;24(suppl_2).

34. Etchi DT, Lilja E, Koponen P, Laatikainen T. Disparities in treatment of diabetes and hypertension among groups of foreign origin and the general Finnish population. Eur J Public Health 2019;29(5),894-899.

35. Howe Hasanali S. Immigrant-Native Disparities in Perceived and Actual Met/Unmet Need for Medical Care. J Immigrant Minority Health 2015;17,1337-1346

36. Brewster A, Curry LA, Cherlin EJ, Talbert-Slagle K, Korwitz LI, Bradley EH. Integrating new practices: a qualitative study of how hospital innovations become routine. Implement Sci 2015;10,168.

37. Mittal S. How organizations implement new practices in dynamic context: role of deliberate learning and dynamic capabilities development in health care units. J. Knowl. Manag. 2019;23(6),1176-1195.

38. Lallukka T, Pietiläinen 0 , Jäppinen S. et al. Factors associated with health survey response among young employees: a registerbased study using online, mailed and telephone interview data collection methods. BMC Public Health 2020;20,184.

39. Christensen Al, Ekholm O, Glümer C, Juel K. Effect of survey mode on response patterns: comparison of face-to-face and selfadministered modes in health surveys. Eur J Public Health 2014;24(2),327-332. 